EPJ Web of Conferences 109,07001 (2016)

DOI: $10.1051 /$ epjconf/201610907001

(C) Owned by the authors, published by EDP Sciences, 2016

\title{
Neutron Star Physics and EOS
}

James M. Lattimer ${ }^{1, a}$

${ }^{1}$ Department of Physics \& Astronomy, Stony Brook University, Stony Brook, NY 11794-3800, USA

\begin{abstract}
Neutron stars are important because measurement of their masses and radii will determine the dense matter equation of state. They will constrain the nuclear matter symmetry energy, which controls the neutron star matter pressure and the interior composition, and will influence the interpretation of nuclear experiments. Astrophysical observations include pulsar timing, X-ray bursts, quiescent low-mass X-ray binaries, pulse profiles from millisecond pulsars, neutrino observations from gravitational collapse supernovae, and gravitational radiation from compact object mergers. These observations will also constrain the neutron star interior, including the properties of superfluidity there, and determine the existence of a possible QCD phase transition.
\end{abstract}

\section{Introduction}

The study of neutron stars plays a vital role in nuclear physics and QCD. Their structure depends in a straightforward way on the dense matter equation of state (EOS). Measurement of their masses, radii, moment of inertia and tidal deformability, to mention just a few global parameters, will tightly constrain the dense matter equation of state, including the properties of the nuclear symmetry energy. They will therefore aid in the interpretation of nuclear experiments and heavy-ion collisions. Among the astrophysical observations that are relevant are pulsar timing, thermal emission from Xray bursters and quisecent low-mass X-ray binaries, temporal variations in emissions from rotating, bursting sources, neutrinos emitted from gravitational collapse supernovae and proto-neutron stars, and gravitational radiation from compact object binaries. These observations will help decipher the composition of the neutron star interior, including the extent and properties of nucleon superfluids, and determine the existence of a possible QCD phase transition. Applications to nuclear physics are therefore supplemented by those to condense matter physics and QCD.

This article summarizes how general relativity and causality, coupled with accurate pulsar-timing mass and rotation measurements, constrain neutron star structure independently of assumptions about the dense matter EOS and other astronomical observations. It will detail how including wellunderstood information about the neutron star's crust, newly determined theoretical calculations of neutron matter, and nuclear structure data concerning the nuclear symmetry energy, allows the tightening of these constraints. It will show how further assumptions concerning the presence of a quarkhadron or other phase transition in the star's interior allows even further limits to be developed. These structure limits will be compared to ongoing astrophysical observations from which mass and radius information can be inferred. The prognosis for further progress from new observations is discussed.

a e-mail: James.Lattimer@Stonybrook.edu 


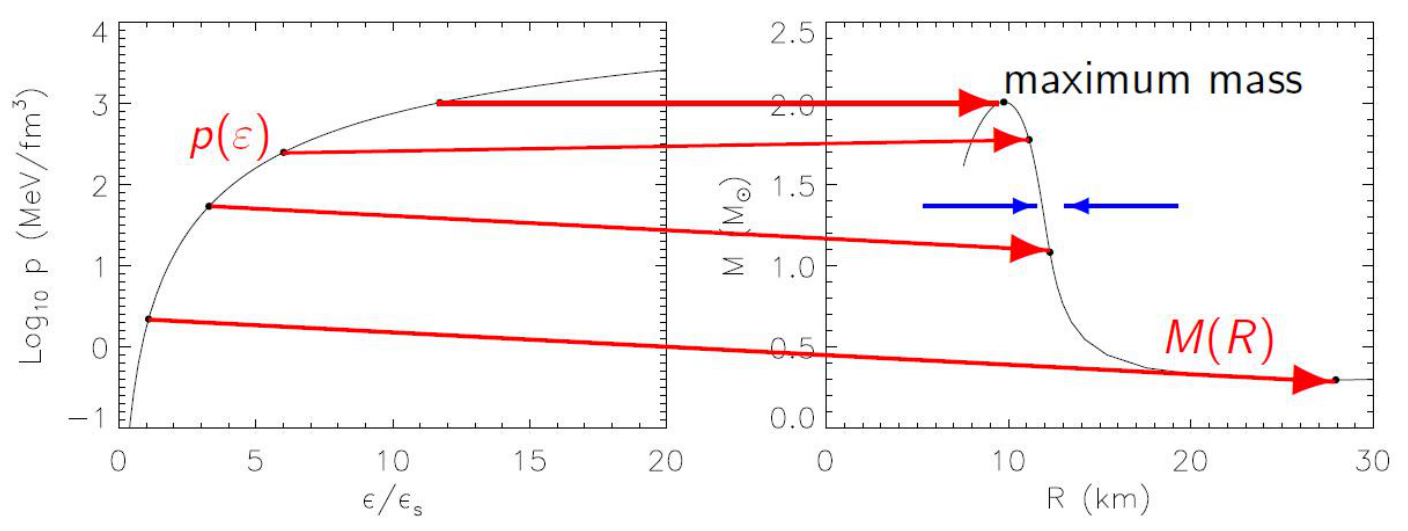

Figure 1. The left panel shows the EOS. Each dot represents the central pressure or energy density which leads to the indicated mass and radius in the right panel showing the full $M-R$ curve. $\epsilon$ is indicated in units of the nuclear saturation density for symmetric matter, $\epsilon_{s} \simeq 150 \mathrm{MeV} \mathrm{fm}^{-3}$. There is a maximum central pressure which leads to a maximum mass; higher central densities lead to gravitational collapse to black holes.

\section{Neutron Star Structure}

The general relativistic equations of stellar structure are

$$
\frac{d p}{d r}=-\frac{G}{c^{4}} \frac{\left(m c^{2}+4 \pi p r^{3}\right)(\epsilon+p)}{r\left(r-2 G m / c^{2}\right)}, \quad \frac{d m}{d r}=4 \pi \frac{\epsilon}{c^{2}} r^{2} .
$$

Here $m(r)$ is the mass interior to radius $r, \epsilon$ is the mass energy density, and $p$ is the pressure. They provide a one-to-one correspondence between the equation of state (EOS, $p(\epsilon)$ ) and the $M-R$ curve, where $M=m(R)$ is the total mass and $R$ is the total radius (Fig. 1). Obvious characteristics of the $M-R$ curve are the existence of a maximum mass and a relatively constant $R$ over a wide mass range from 1 to $1.8 M_{\odot}$. The former is a consequence of the factor $r-2 G m / c^{2}$ in the denominator of Eq. (1): as the compactness $m / r$ increases, the pressure gradient required for stability grows. Even an infinite pressure won't stabilize sufficiently compact and massive configurations. The latter feature can be understood by ignoring relativity altogether and dimensionally analyzing these equations. Assuming the polytropic relation $p=K \epsilon^{\gamma}$ for the EOS throughout a star, one finds the mass-radius relation

$$
M=Q(\gamma) K^{1 /(2-\gamma)} R^{(4-3 \gamma) /(2-\gamma)},
$$

where $Q$ is a constant. We therefore find for low-density stars that $M \propto K^{3 / 2} R^{0}$, or the total mass $M$ is independent of the stellar radius $R$. Indeed, the mass approaches the so-called minimum neutron star mass, about $0.1 M_{\odot}$ as the compactness $\beta=G M / R c^{2} \rightarrow 0$. Above the nuclear saturation density $\epsilon_{s}$, realistic EOSs have $\gamma \simeq 2$, for which $R \propto K^{1 / 2} M^{0}$, or the radius is independent of the mass. This holds until $\beta$ becomes large (i.e., $\beta \gtrsim 0.1$ ) and general relativity can no longer be ignored. In both cases, the value of $K$ is quantitatively important in determining either the limiting mass or radius. These features are apparent in the $M-R$ curve displayed in Fig. 1.

The maximum compactness is a relevant quantity, as it determines the minimum radius as a function of mass. While an extreme limit from Eq. (1) appears to be $\beta=1 / 2$, a more restrictive limit follows from the analytic solution of these equations in the case that $\gamma \rightarrow \infty$, the incompressible case for which $\epsilon=\epsilon_{c}=3 M /\left(4 \pi R^{3}\right)$, a constant. In this case, the pressure in the star's interior is

$$
p(r)=\frac{3 \beta c^{4}}{4 \pi G R^{2}} \frac{\sqrt{1-2 \beta(r / R)^{2}}-\sqrt{1-2 \beta}}{3 \sqrt{1-2 \beta}-\sqrt{1-2 \beta(r / R)^{2}}} .
$$


The denominator vanishes at the star's center for $\beta=4 / 9$, but causality requires that $p_{c}=p(r=$ $0)<\epsilon_{c}$, which implies $\beta<3 / 8$. This holds for any star for which $d \epsilon / d r \leq 0$ for all $r$ [1]. However, the maximally-compact conjecture[2] leads to the condition $\beta<0.354$ [3]. This conjecture is that the maximum compactness, while ensuring causality, is achieved for the EOS $p=\epsilon-\epsilon_{o}$ for $\epsilon \geq \epsilon_{o}$, and $p=0$ otherwise. At this limit, the maximum mass is achieved, and one finds the central energy density and pressure are $\epsilon_{c}=3.034 \epsilon_{o}$ and $p_{c}=2.034 \epsilon_{o}$, respectively[4]. Also, the maximum mass is

$$
M_{\max }=0.0852 \frac{c^{4}}{\sqrt{G^{3} \epsilon_{0}}} \simeq 4.09 \sqrt{\frac{\epsilon_{s}}{\epsilon_{0}}} M_{\odot} .
$$

This result can be inverted to yield a maximum value for $\epsilon_{0}$ based on the largest accurately measured neutron star mass, which is currently about $M_{\max }=1.97 M_{\odot}$ [5]. In turn, we immediately obtain the maximum pressure or energy density in any neutron star

$$
\epsilon_{c, \text { max }}=3.034 \epsilon_{0, \text { max }} \simeq 50.8 \epsilon_{s}\left(\frac{M_{\odot}}{M_{\max }}\right)^{2}, \quad p_{c, \text { max }}=2.034 \epsilon_{0, \text { max }} \simeq 34.1 \epsilon_{s}\left(\frac{M_{\odot}}{M_{\max }}\right)^{2} .
$$

The discovery of a star with $M>1.97 M_{\odot}$ would decrease these limits. The $M-R$ curve for the maximally-compact EOS, which is the solution to the structure equations scaled to achieve $M_{\max }$, then establishes a boundary of minimum radii $R$ as function of stellar mass $M$. This is displayed in Fig. 2. Solutions assuming a limiting squared sound speed $s=1 / 3$ are also shown, which illustrate minimum radii for stars largely made from deconfined quark matter.

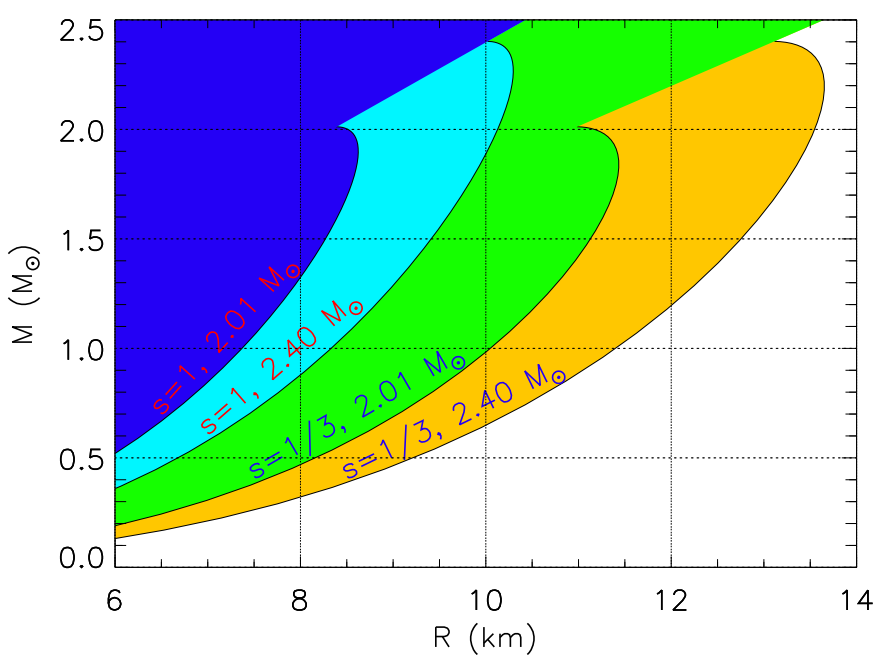

Figure 2. Mass-radius diagram showing regions excluded by causality and the maximally compact conjecture. Boundaries are indicated for $M_{\max }=2.01 M_{\odot}$ and $2.4 M_{\odot}$ and for sound speed squared $s=1$ and $1 / 3$.

The maximally compact conjecture is extreme in that it ignores the existence of a neutron star crust and the finite pressure of matter in the vicinity of the saturation density $n_{s} \simeq 0.16 \mathrm{fm}^{-3}$ or $\rho_{s} \simeq$ $2.7 \times 10^{14} \mathrm{~g} \mathrm{~cm}^{-3}$. Observations of pulsar glitches, neutron star cooling following transient heating events, and observed thermal emissions from cooling neutron stars favor the existence of a crust as opposed to a large surface density as would be the case for a pure strange quark star (sometimes called a self-bound star). The pressure in the crust is largely due to relativistic, degenerate electrons with at 
most a 5\% contribution from nuclei and neutrons[6]. The major contribution of baryons to the pressure is from the collective Coulomb pressure due to the nuclear lattice, and is therefore largely independent of uncertainties in the EOS of nuclear matter. Matter in neutron stars is in beta equilibirum, i.e., the energy is minimized with respect to proton concentration, leading to $\partial \epsilon / n / \partial x=\mu_{n}-\mu_{p}-\mu_{e}=0$, where $x=n_{p} / n$. As a consequence, the crust becomes progressively more neutron rich with increasing density due to the increase in $\mu_{e}=\hbar c(3 \pi n x)^{1 / 3}$ with density.

Various calculations indicate that the transition from crustal material to uniform nuclear matter occurs in the range $n_{0} \approx n_{s} / 4-n_{s} / 2$. We will assume the crust-core transition density is $n_{0}=n_{s} / 2.7$, and use the BPS [6] crustal EOS, for which the pressure, energy density and internal energy per baryon at $n_{0}$ are $p_{0}=0.243 \mathrm{MeV} \mathrm{fm}^{-3}, \epsilon_{0}=56.39 \mathrm{MeV} \mathrm{fm}^{-3}$, and $E_{0}=12.13 \mathrm{MeV}$. Above this density, matter is a structureless uniform fluid.

The energy difference of uniform matter between pure neutron $(x=0)$ and symmetric $(x=1 / 2$ matter is the symmetry energy $S(n)$. It appears that matter with intermediate values of $x$ can be described with a quadratic interpolation:

$$
E(n, x) \simeq E(n, 1 / 2)+S(n)(1-2 x)^{2},
$$

$E\left(n_{s}, 1 / 2\right)=-B \simeq-16 \mathrm{MeV}$. A convenient estimate for the symmetry energy near $n_{s}$ is

$$
S(n)=S_{v}\left(n / n_{s}\right)^{\gamma},
$$

with $S_{v}=S\left(n_{s}\right)$ Nuclear experimental information and neutron matter calculations indicate that $26 \mathrm{MeV} \lesssim S_{v} \lesssim 34 \mathrm{MeV}$ and $0.3 \lesssim \gamma \lesssim 0.7$, with the value of $\gamma$ positively correlated with $S_{v}$. The corresponding liquid droplet parameter $L=3(n d S / d n)_{n_{s}}$ is $3 S_{v} \gamma$ which is then $23 \mathrm{MeV} \lesssim L \lesssim 71 \mathrm{MeV}$. This range is nicely corroborated by experimental studies of nuclear binding energies, dipole resonances, and neutron skin thicknesses[7].

The pressure corresponding to Eqs. (6) and (7) is

$$
p(n, x)=n^{2} \frac{\partial E(n, x)}{\partial n} \simeq p(n, 1 / 2)+S_{v} \gamma n_{s}\left(\frac{n}{n_{s}}\right)^{\gamma+1}(1-2 x)^{2} .
$$

By definition, $p\left(n_{s}, 1 / 2\right)=0$; to leading order, near $n_{s}$, the symmetric matter pressure increases linearly with density, $p(n, 1 / 2) \simeq\left(K_{s} / 9\right)\left(n-n_{s}\right)$, where $K_{s} \simeq 240 \mathrm{MeV}$ is the incompressibility parameter. Beta equilibrium determines $x$ as a function of density $n$, and is approximately

$$
x_{\beta} \simeq \frac{64 S_{v}^{3}\left(n / n_{s}\right)^{3 \gamma}}{3 \pi^{2}(\hbar c)^{3}+128 S_{v}^{3}\left(n / n_{s}\right)^{3 \gamma}}
$$

which has the value $x_{\beta} \simeq 0.04$ when $n=n_{s}$. Neutron matter pressure at saturation, using Eq. (7), is $p\left(n_{s}, 0\right)=\gamma n_{s} S_{v}$. Neutron star matter pressure is then

$$
p\left(n_{s}, x_{\beta}\right) \simeq \gamma n_{s} S_{v}\left[1-\left(\frac{4 S_{v}}{\hbar c}\right)^{3} \frac{4 \gamma-1}{3 \pi^{2} \gamma}\right] .
$$

The correction term in Eq. (10) is of order 1.4\%, and can be ignored to good approximation.

Lattimer \& Prakash[7, 8] found the phenomenological quasi-universal correlations between the radii of $1.4 M_{\odot}$ stars and neutron star matter pressure, a consequence of Eq. (2):

$$
R_{1.4}=(9.52 \pm 0.49)\left[p\left(n_{s}, x_{\beta}\right)\right]^{1 / 4} \mathrm{~km} ; R_{1.4}=(5.68 \pm 0.14)\left[p\left(2 n_{s}, x_{\beta}\right)\right]^{1 / 4} \mathrm{~km} .
$$


It serves as the fundamental connection between nuclear physics and neutron star structure. Another relation concerns the maximum spin frequency $f$ of a neutron star. The mass-shedding limit occurs when $f=f_{K}$, the Keplerian frequency phenomenologically determined to be[9, 10]

$$
f_{K}=1.08\left(\frac{M}{M_{\odot}}\right)^{1 / 2}\left(\frac{10 \mathrm{~km}}{R}\right)^{3 / 2} \mathrm{kHz}
$$

where $M$ and $R$ refer to the non-spinning star. The highest frequency pulsar known is PSR J17482446ad[11] with $f=716 \mathrm{~Hz}$, so $f_{K} \geq f$ and $M \leq 2 M_{\odot}$ imply a limiting radius

$$
R \lesssim 10.5\left(\frac{\mathrm{kHz}}{f}\right)^{2 / 3}\left(\frac{M}{M_{\odot}}\right)^{1 / 3} \mathrm{~km} \lesssim 16.6 \mathrm{~km} .
$$

For densities above the core-crust transition density, we employ piecewise polytropic regions with continuous pressures at the boundaries. Each region $i\left(n_{i-1}<n<n_{i}\right)$ has the EOS $p=n_{i-1}\left(n / n_{i-1}\right)_{i}^{\gamma}$, motivated by Ref. [12], who found a 3-polytrope scheme could match, to about $4 \%$ rms error, the pressure-energy density relations for a large set of candidate EOSs below the central densities of $1.4 M_{\odot}$ stars, including exotic EOSs with kaons or hyperons. The starting density is the core-crust transition density $n_{0}$, and the other boundaries are fixed at $n_{1}=1.85 n_{s}$ and $n_{2}=3.7 n_{s}$, respectively. The $\operatorname{EOS} \epsilon(p)$ in region $i$ is easily found:

$$
\epsilon(p)=\left(\epsilon_{i-1}-\frac{p_{i-1}}{\gamma_{i}-1}\right)\left(\frac{p}{p_{i-1}}\right)^{1 / \gamma_{i}}+\frac{p}{\gamma_{i}-1}, \quad \gamma_{i}=\frac{\ln \left(p_{i} / p_{i-1}\right)}{\ln \left(n_{i} / n_{i-1}\right)},
$$

where $\epsilon_{i-1}=n_{i-1}\left(m_{n}+E_{i-1}\right)$ and $E_{i-1}$ is energy density and energy per baryon at the point $n_{i-1}$. For densities $n>n_{3}$ matter is treated as a continuation of region 3 . We will also consider a model in which the densities $n_{1}$ and $n_{2}$ are varied, so as to explore the possibility of strong phase transitions near $n_{s}$.

The parameters of the first scheme are thus $p_{1}, p_{2}$ and $p_{3}$, supplemented by $n_{1}$ and $n_{2}$ in the second scheme. Neutron matter studies sets the limit $7.3 \mathrm{MeV} \mathrm{fm}^{-3} \leq p_{1} \leq 21 \mathrm{MeV} \mathrm{fm}^{-3}[13,14]$, while $p_{1} \leq p_{2} \leq p_{2 c}$ and $p_{2} \leq p_{3} \leq p_{3 c}$ are limits from stability and causality. Here, the causal limits, indicated by subscript $c$, are determined by

$$
\left(\frac{\partial p}{\partial \epsilon}\right)_{i c}=\frac{\gamma_{i c} p_{i c}}{\epsilon_{i c}+p_{i c}}=1
$$

which is an implicit equation for $p_{i c}$ given Eq. (14). Some parameter combinations can still be disallowed if the maximum mass configurations have central pressures $p_{c}>p_{3}$ because even though the EOS is causally limited at $n_{3}$, causality could be violated at larger densities.

For the first model, it is found that causal configurations capable of support $M_{\max } \simeq 1.97 M_{\odot}$ must have $100 \mathrm{MeV} \mathrm{fm}^{-3} \lesssim p_{2} \lesssim 250 \mathrm{MeV} \mathrm{fm}^{-3}$ and $190 \mathrm{MeV} \mathrm{fm}^{-3} \lesssim p_{3} \lesssim 1300 \mathrm{MeV} \mathrm{fm}^{-3}$. The lower limit of $p_{2}$ is independent of $p_{1}$ but its upper limit increases with $p_{1}$. The lower limit to $p_{3}$ is $p_{2}$ and its upper limit is also approximately linearly correlated with the value of $p_{2}$. There is a strong correlation between $M_{\max }$ and $p_{2}$, but only a weak correlation between $M_{\max }$ and $p_{3}$. It is also found that $R_{1.4}$ is highly correlated with both $p\left(n_{s}\right)$ and $p_{1}$, in accordance with Eq. (11), but not with $p_{2}$ or $p_{3}$. The overall constraints on the EOS and the allowed $M-R$ region are shown in Fig. 3. The allowed regions sensitively depend on the assumed $M_{\max }$. Note for $M_{\max }=1.97 M_{\odot}$ that $10.7 \mathrm{~km} \lesssim R_{1.4} \lesssim 13.1 \mathrm{~km}$.

The case of variable densities $n_{1}$ and $n_{2}$ allows for strong phase transitions and widens the allowed radius range. The extreme cases are when $n_{1}=n_{s}, n_{2}=4 n_{s}$. and $\gamma_{2}=0$, for which the upper limit increases to $14.3 \mathrm{~km}$, and when $n_{1}=n_{2}=n_{s}$ and $\gamma_{2}=0$ for which the lower limit decreases to $8.4 \mathrm{~km}$, about $0.3 \mathrm{~km}$ larger than the maximally compact limit. Realistically, the radius range is only moderately broadened, as it is unlikely a phase transition occurs close to $n_{s}$. It is also interesting to find phase transitions are excluded if $M_{\max } \gtrsim 1.97 M_{\odot}$ and $n_{1} \gtrsim 2.03 n_{s}$. 


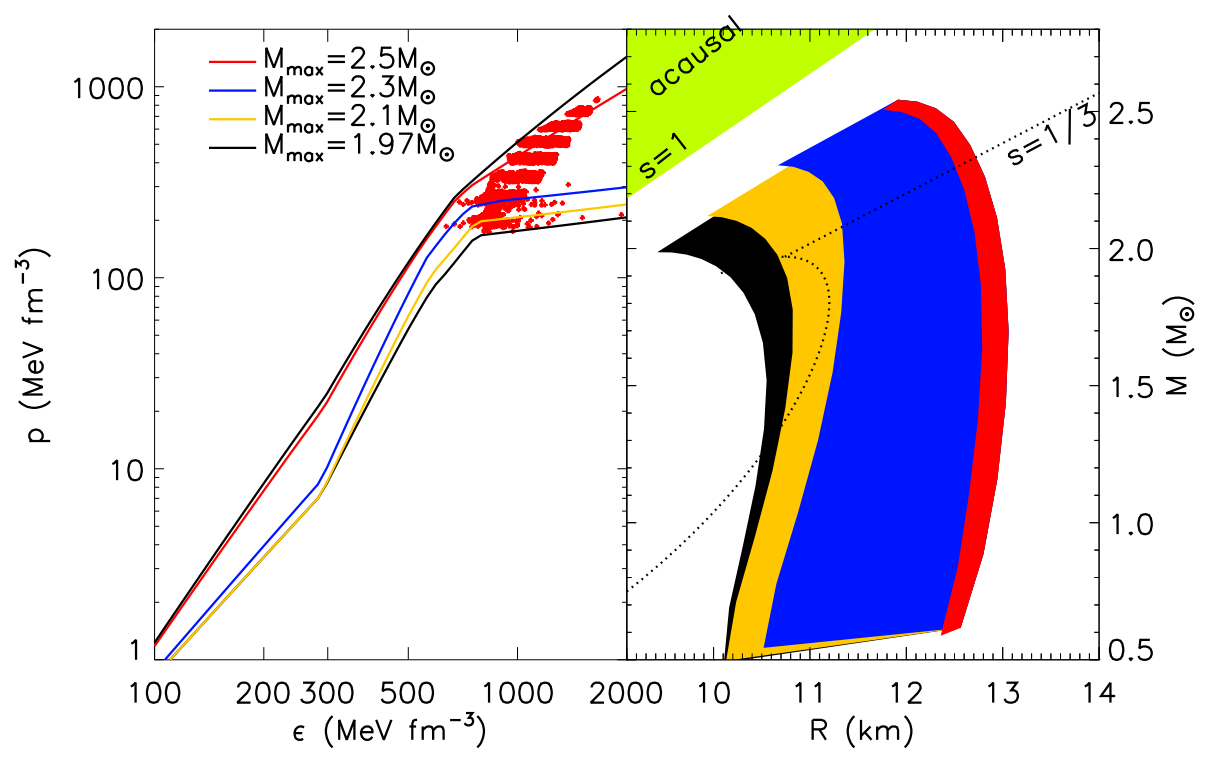

Figure 3. Left panel: Allowed pressures as a function of energy density permitted by the assumed constraints on the low-density EOS, causality, and selected values for $M_{\max }$. Red crosses indicate the central conditions for surviving EOSs. The black, yellow, blue and red lines are for $M_{\max }=1.97 M_{\odot}, 2.1 M_{\odot}, 2.3 M_{\odot}$, and $2.5 M_{\odot}$, respectively. Right panel: Allowed masses and radii for selected values of $M_{\max }$. Figure from Ref. [15].

\section{Observational Constraints on Radii}

The best current observational radius constraints originate from thermal emissions from photospheric radius expansion (PRE) Type I X-ray bursts and from quiescent low-mass X-ray binaries (qLMXBs). In the case of PRE bursts, two observational parameters can be identified:

$$
F_{E d d, \infty}=\frac{G M c}{\kappa D^{2}} \sqrt{1-2 \beta}, \quad A=\frac{F_{\infty}}{\sigma_{B} T_{e f f, \infty}^{4}}=f_{c}^{-4}\left(\frac{R_{\infty}}{D}\right)^{2} .
$$

$F_{E d d}$ is the Eddington flux, observed as the peak burst flux, and $A$ is a normalization related to the angular area. $\kappa$ is the opacity, $F$ and $T_{\text {eff }}$ are the flux and effective temperature on the cooling tail. The subscript $\infty$ indicates the quantites are redshifted as observed on Earth. $f_{c}=T_{c} / T_{\text {eff }}$ contains the effects of the atmosphere, where $T_{c}$ is the color temperature. The observables can be combined into two parameters

$$
\alpha=\frac{F_{E d d, \infty}}{\sqrt{A}} \frac{\kappa D}{f_{c}^{2} c^{3}}=\beta(1-2 \beta), \quad \gamma=\frac{A}{F_{E d d, \infty}} \frac{f_{c}^{4} c^{3}}{\kappa}=\frac{R}{\alpha \sqrt{1-2 \beta}} .
$$

Real solutions for $R$ exist if $\alpha \leq 1 / 8$. For 6 bursters examined by Özel and collaborators[16], $0.17 \leq$ $\alpha \leq 0.21$ and $68 \mathrm{~km}<\gamma<90 \mathrm{~km}$, so real solutions don't exist. Since observational and theoretical uncertainties in $\alpha$ and $\gamma$ are about 20\%, one can Monte Carlo sample over their error distributions. Only about $4 \%$ of the trials give real solutions, indicating a serious inconsistency between the model and observations exists. The preferred values of $\alpha$ and $\gamma$ are near $1 / 8$ and between $100 \mathrm{~km}$ and 130 $\mathrm{km}$, respectively. Preferred radius values are between 8 and $10 \mathrm{~km}$. 
Two alternatives have been proposed to remedy the inconsistency. Ref. [17] suggested that the peak flux is observed when the photosphere is still levitated far above the surface, so the redshift factor $\sqrt{1-2 \beta}$ is missing from $F_{E d d}$. In this case, $\alpha=\beta \sqrt{1-2 \beta}$ so that $\alpha \leq 3^{-3 / 2} \simeq 0.192$ is required for real solutions. Now about $65 \%$ of Monte Carlo trials yield real solutions, with $\alpha \simeq 0.18$ and $70 \mathrm{~km}$ $<\gamma<90 \mathrm{~km}$ being preferred. Radii in the range of $10 \mathrm{~km}$ to $12 \mathrm{~km}$ are then preferred.

Another possibility is that the bursts studied by Özel et al. are incompatible with the simple model proposed for them, but different sets of bursts, so-called 'passive' bursts, may avoid the uncertainty of the photospheric radius and color correction $f_{c}$ completely. In addition, these bursts may be studied using information from the whole cooling tail rather than just near the peak and at late times. Nättilä et al.[18] recently performed a study of 3 sources with this cooling tail method and showed that the parameters $F_{E d d, \infty}$ and $A^{\prime}=D / R_{\infty}=1 / \sqrt{f_{c}^{4} A}$ could be determined to about $3 \%$ and $1 \%$ accuracy, respectively. The 3 sources have nearly identical values of $\alpha=0.117$ and $\gamma=128 \mathrm{~km}$ to about $2 \%$. Uncertainties in $\alpha$ are dominated by the distance and are about $10 \%$, while uncertainties in $\gamma$ are less than $4 \%$. Nearly all Monte Carlo trials are accepted, and preferred values of $\beta, M$ and $R$ are $0.185,1.5 M_{\odot}$ and $12 \mathrm{~km}$, respectfully, for all 3 sources.

The situation for qLMXBs is plagued by uncertainties in the amount of absorbing material between them and Earth and the compositions of the stars' atmospheres. A study by Guillot et al.[19] found individual radii for 5 sources ranged from $7 \mathrm{~km}$ to $20 \mathrm{~km}$, but a combined analysis under the assumption that all sources must have a common radius yielded $R \simeq 9.0 \pm 1.4 \mathrm{~km}$. Ref. [20] concluded that different, but independently derived, interstellar absorptions and the possibility that some sources have helium, rather than hydrogen, atmospheres would resolve the large radius range conundrum, yielding a smaller range $8 \mathrm{~km}<R<11 \mathrm{~km}$ for the central values of the 5 sources. Nevertheless each source still had 20-30\% uncertainties. These conclusions were confirmed by Ref. [21].

A novel approach to determining neutron star parameters from observations was a Bayesian approach utilizing constraints on the allowed masses and radii from priors stemming from EOS, causality and general relativistic considerations[17]. Without observational data, these priors predict radius ranges similar to those from the piecewise polytrope analysis above, $10.7 \mathrm{~km}<R_{1.4}<13.1 \mathrm{~km}$. Observational data from both PRE bursts and qLMXBs tend to favor a range with a smaller mean value, $10.7 \mathrm{~km}<R_{1.4}<12.5 \mathrm{~km}$, even including the possibility of strong phase transitions.

\section{Conclusions}

Our understanding of neutron star structure has advanced greatly in the last decade. The minimum value for the maximum mass has increased from about $1.5 M_{\odot}$ to $2 M_{\odot}$, and the uncertainties in typical radii have shrunk from over $5 \mathrm{~km}$ to about $2 \mathrm{~km}$. Theoretical neutron matter calculations, the interpretation of experimental nuclear data, and the interpretation of astrophysical observations all yield very nearly the same ranges for neutron star radii. Other advances, only partly discussed here, include the discovery of quasi-universal (i.e., EOS independent) correlations between structural properties such as the compactness $\beta$, the binding energy, the moment of inertia and the tidal deformability which will greatly aid the interpretation of future obsevations from radio, X-rays and gravitational radiation.

Among the interesting prospects for further determinations of neutron star structure include:

- The moment of inertia of the pulsar PSR J0737-3039A from observations of periastron advance[22]. In principle, this has little systematic uncertainty.

- Periodic emissions from spinning neutron stars using pulse profiles in multiple wavebands[24, 25]. This method is independent of distance and interstellar absorption which plague PRE burst and qLMXB studies. The NICER mission[23], planned for August 2016, is devoted to this method. 
- Gravitational radiation by Advanced LIGO and VIRGO from merging compact objects[26]. Tidal effects during inspiral offer the possibility of breaking the mass degeneracy in the observed chirp mass $\mathcal{M}=\left(M_{1} M_{2}\right)^{3 / 5} / M^{1 / 5}$ through the measurement of the tidal deformability $\lambda[27]$. In addition, both merger and postmerger waveforms from black hole-neutron star and neutron star-neutron star have peak frequencies that correlate with either the compactness $\beta$ or $\bar{\lambda}=\lambda / M^{5}$ in a nearly universal way[28]. This is important, given an extremely strong universal correlation between $\bar{\lambda}$ and $I$ [29].

\section{References}

[1] S. Weinberg, Gravitation and Cosmology (John Wiley \& Sons, Inc., New York 1972), §11.6

[2] S. Koranda, N. Stergioulas and J.L. Friedman, Ap. J. 488, 799 (1997)

[3] P. Haensel, J.P. Lasota and J. L.Zdunik, A \& A 344, 13 (1999)

[4] J.M. Lattimer and M. Prakash, in From Nuclei to Neutron Stars, ed. S. Lee (World Scientific, Singapore, 2011) 275

[5] P.B. Demorest et al., Nature 467, 1081 (2010); J. Antoniadis et al., Science 340, 448 (2013)

[6] G. Baym, C.J. Pethick and P. Sutherland, Ap. J. 170, 299 (1971)

[7] J.M. Lattimer and Y. Lim, Ap. J. 771, 51 (2013)

[8] J.M. Lattimer and M. Prakash, Ap. J. 550, 426 (2001)

[9] J.M. Lattimer and M. Prakash, Science 304, 536 (2004);

[10] P. Haensel, J.L. Zdunik, M. Bejger and J.M. Lattimer, Ast. Ap. 502, 605 (2009)

[11] J.W.T. Hessels et al., Nature 311, 1901 (2006)

[12] J.S. Read, B.D. Lackey, B.J. Owen and J.L. Friedman, Phys. Rev. D 79, 124032 (2009)

[13] K. Hebeler, J.M. Lattimer, C.J. Pethick and A. Schwenk, Ap. J. 773, 11 (2013);

[14] S. Gandolfi, J. Carlson and S. Reddy, Phys. Rev. C 84, 032001 (2012)

[15] J.M. Lattimer and M. Prakash, Phys. Rep., submitted (2015)

[16] F. Özel et al., arXiv:1505.05155 (2015)

[17] A.W. Steiner, J.M. Lattimer and E. Brown, Ap. J. 722, 33 (2010)

[18] J. Nättilä et al., arXiv:1409.06561 (2015)

[19] S. Guillot, M. Servillat, N.A> Webb and R.E. Rutledge, Ap. J. 772, 7 (2013)

[20] A.W. Steiner, J.M. Lattimer and E. Brown, Ap. J. Lett. 765, L5 (2013)

[21] C.O. Heinke et al., MNRAS 444, 443 (2014)

[22] J.M. Lattimer and B.F. Schutz, Ap. J. 629, 979 (2005)

[23] K. Gendreau, Z. Arzoumanian and T. Okajima, SPIE 8443, 13 (2012)

[24] D. Psaltis, F. Özel and D. Chakrabarty, Ap. J. 707, 136 (2014);

[25] M.C. Miller and F.K. Lamb, Ap. J. 808, 31 (2015)

[26] J. Abadie et al., Class. Quant. Grav. 27, 173001 (2010)

[27] E.E. Flanagan and T. Hinderer, Phys. Rev. D 77, 021502 (2008)

[28] J. S. Read et al., Phys. Rev. D 88, 044042 (2013); A. Bauswein, N. Stergioulas and H.-T. Janka,

Phys. Rev. D 90, 023002 (014); S. Bernuzzi, T. Dietrich and A. Nagar, Phys. Rev. Lett. 115, 091101

(2015); K. Takami, L. Rezzolla and B. Luca, J. Ph.: Conference Series 600, 012056 (2015)

[29] K. Yagi and N. Yunes, Phys. Rev. D 88, 023009 (2013) 